\title{
PONTO DE ENTRADA PARA AS HASTES INTRAMEDULARES ANTERÓGRADAS DO FÊMUR: ESTUDO EM CADÁVER
}

\section{ENTRY POINT FOR THE ANTEROGRADE FEMUR INTRAMEDULLARY NAIL:} A CADAVER STUDY

Pedro José Labronici', Luiz Galeno², Thiago Martins Teixeira², José Sergio Franco ${ }^{3}$, Rolix Hoffmann 4 , Paulo Roberto Barbosa de Toledo Lourenço ${ }^{5}$, Vincenzo Giordano ${ }^{6}$, Alexandre Pallottino ${ }^{7}$, Ney Pecegueiro do Amaral ${ }^{8}$

\section{RESUMO}

Objetivo: Analisar a saída natural do fio-guia no trocânter maior pela via retrógrada do fêmur, em espécimes de cadáver. Métodos: 100 fêmures foram perfurados entre os côndilos femorais, a $1,2 \mathrm{~cm}$ da região intercondilar. Um fio-guia reto de $3 \mathrm{~mm}$ foi introduzido, de forma retrógrada, até alcançar a extremidade proximal do fêmur. Foram avaliados em relação à região posterossuperior e anterossuperior do trocânter maior, fossa piriforme e linha mediana superior entre a cabeça-colo e trocânter maior. Resultados: Em $62 \%$ o fio-guia reto saiu na face anterior do trocânter maior. Na fossa piriforme, a distância mediana observada foi de $1,0 \mathrm{~cm}$ e a amplitude interquartílica, de $0,5 \mathrm{~cm}$, expressando inicialmente, em relação à fossa piriforme, melhor precisão. Conclusão: O eixo central do canal medular, na incidência coronal, projetou melhor precisão na região da fossa piriforme.

Descritores - Fêmur; Fixação intramedular de fraturas; Cadáver

\section{ABSTRACT}

Objective: To analyze the natural exit of the wire guides in major trochanter through retrograde femoral approach, in cadaver specimens. Material and Method: 100 femurs had been perforated between the femoral condyles, at $1.2 \mathrm{~cm}$ of the intercondylar region. A 3-mm straight wire guide was introduced, through retrograde approach, until the proximal extremity of femur was reached. Femurs were assessed for posterosuperior and anterosuperior portions of major trochanter, pear-shaped cavity, and upper median line between the head-neck and the major trochanter. Results: in 62\%, the straight wire guides exited at the anterior surface of major trochanter. In the pear-shaped cavity, the median distance found was $1.0 \mathrm{~cm}$ and the interquartile range was $0.5 \mathrm{~cm}$, initially expressing, in relation to pear-shaped cavity, better accuracy. Conclusion: the central axis of the medullar canal, at coronal plane, projected better accuracy in the region of the pear-shaped cavity.

Keywords - Femur; Fracture fixation; intramedullary; Cadaver

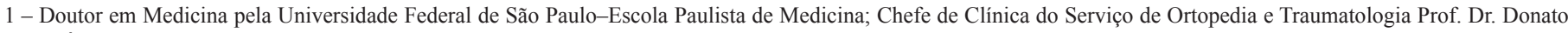
D’Ângelo - Hospital Santa Teresa, Petrópolis/RJ, Brasil.

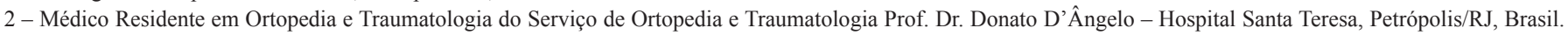

3 - Professor Associado do Departamento de Ortopedia e Traumatologia da Faculdade de Medicina da Universidade Federal do Rio de Janeiro/RJ, Brasil.

4 - Médico do Serviço de Ortopedia e Traumatologia Prof. Dr. Donato D’Ângelo - Hospital Santa Teresa, Petrópolis/RJ, Brasil.

5 - Médico Responsável pelo Grupo de Trauma do Hospital de Ipanema, Rio de Janeiro, Brasil.

6 - Coordenador do Programa de Residência Médica do Hospital Municipal Miguel Couto, Rio de Janeiro, Brasil.

7 - Médico Ortopedista do Serviço de Ortopedia e Traumatologia do Hospital Municipal Miguel Couto, Rio de Janeiro, Brasil.

8 - Chefe do Serviço de Ortopedia e Traumatologia do Hospital Municipal Miguel Couto, Rio de Janeiro, Brasil. 


\section{INTRODUÇÃO}

Para se obter sucesso quando se utiliza a técnica da haste intramedular anterógrada para o tratamento das fraturas do fêmur, além de se ter um bom conhecimento da anatomia da extremidade proximal do fêmur, deve-se saber escolher o ponto de entrada correto para introdução da haste. $\mathrm{O}$ objetivo principal em definir o ponto de entrada é conseguir um alinhamento anatômico dos fragmentos ósseos.

Existem diferentes opiniões na literatura sobre o melhor local do ponto de entrada na extremidade proximal do fêmur. Alguns autores preferem a ponta do trocânter maior $^{(1-3)}$. Outros preferem a fossa piriforme, pois acreditam que esta localização seria o eixo entre o trocânter e a diáfise femoral ${ }^{(4-7)}$. Também têm sido descritas as regiões do terço anterior e dois terços posteriores da ponta do trocânter maior ${ }^{(8,9)}$.

O objetivo deste trabalho foi analisar a saída natural do fio-guia no trocânter maior pela via retrógrada do fêmur, em espécimes de cadáver.

\section{MÉTODOS}

Foram utilizados 100 fêmures da Faculdade de Medicina de Petrópolis/RJ. Foram excluídos os fêmures com deformidades prévias, sinais de fraturas ou desgaste do trocânter maior e/ou côndilo femoral. Dos espécimes anatômicos, 47 eram do lado direito e 48 do esquerdo. Todos os espécimes femorais foram perfurados com uma broca de $6 \mathrm{~mm}$ entre os côndilos femorais, a 1,2cm da região intercondilar ${ }^{(10,11)}$. Um fio-guia reto de $3 \mathrm{~mm}$ foi introduzido, de forma retrógrada, até alcançar a extremidade proximal do fêmur. Cinco fêmures foram excluídos devido a fraturas do trocânter maior durante o procedimento de passagem do fio-guia, totalizando 95 espécimes anatômicos. O local de saída do guia foi medido em relação à região posterosuperior (PST) e anterossuperior do trocânter maior (AST), fossa piriforme (FP) e linha mediana superior entre a cabeça-colo e trocânter maior (Figuras 1 e 2).

\section{RESULTADOS}

Este estudo teve por finalidade traçar um perfil da frequência do fio-guia na região trocantérica, após introdução por via retrógrada na região intercondilar do fêmur em 100 espécimes anatômicos. Foram descartados cinco fêmures, pois ao introduzir o fio-guia houve fratura do trocânter maior, o que impossibilitou a tomada das medidas.

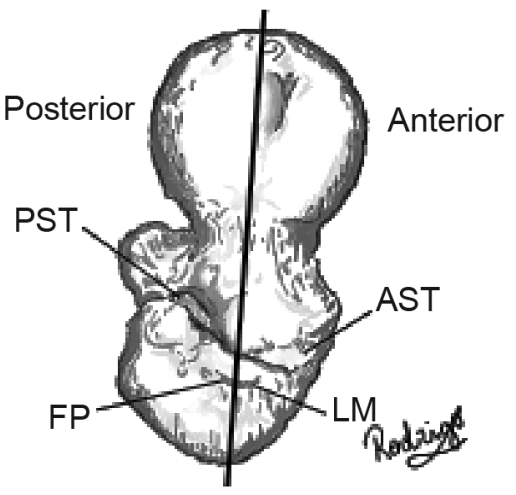

Figura 1 - Visão superior da extremidade proximal do fêmur. PST - região posterossuperior do trocânter maior; AST - região anterossuperior do trocânter; FP - fossa piriforme; e LM - linha média da extremidade proximal do fêmur.

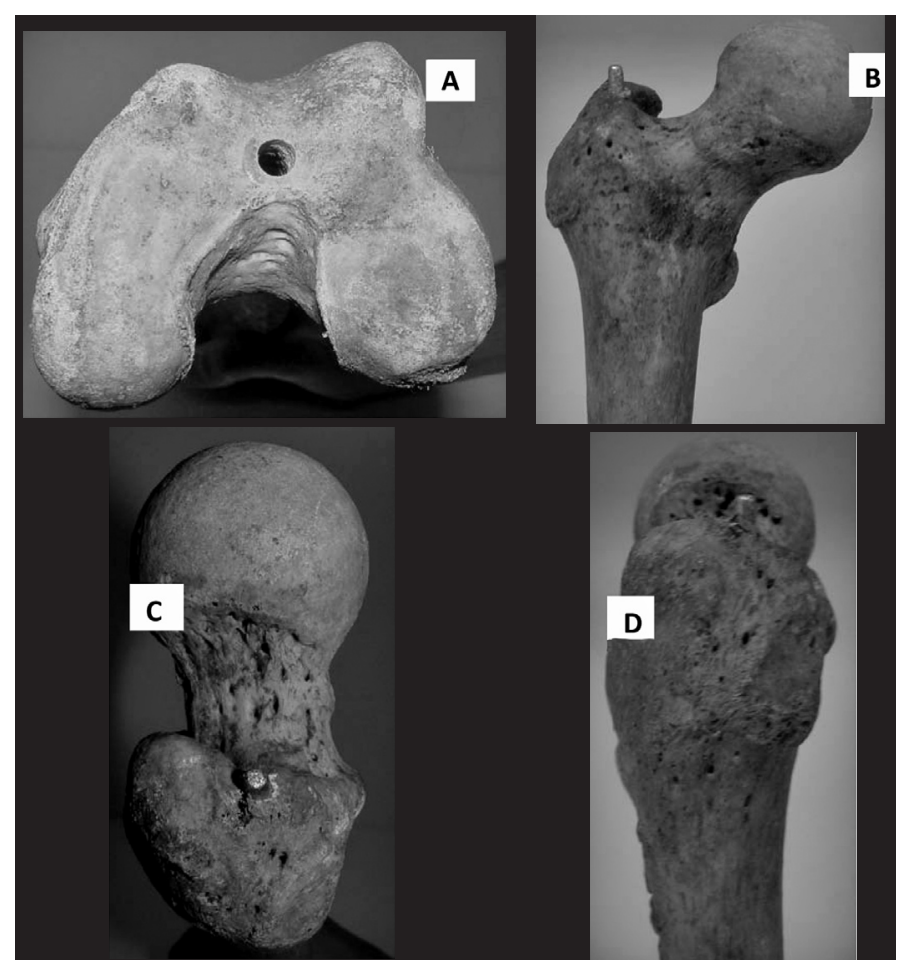

Figura 2 - A) Localização da entrada do fio-guia na região intercondiliana; B) Visão em anteroposterior da extremidade proximal do fêmur com saída do fio-guia. C) Visão superior da extremidade proximal do fêmur com saída do fio-guia. D) Visão lateral da extremidade proximal do fêmur com saída do fio-guia.

Este trabalho foi analisado e aprovado pelo Comitê de Ética da Faculdade de Medicina de Petrópolis.

A tabela 1 fornece as medidas de tendência central e de dispersão das distâncias em relação ao ponto de entrada retrógrado de 95 fêmures com média, desvio padrão (DP), moda, $1^{\circ}$ quartil, $2^{\circ}$ quartil (mediana), $3^{\circ}$ quartil, percentil de $10 \%$, percentil de $90 \%$, mínimo e máximo. 
Tabela 1 - Descritiva das distâncias $(\mathrm{cm})$ em relação ao ponto de entrada retrógrado

\begin{tabular}{|c|c|c|c|c|c|c|c|c|c|c|c|}
\hline Distância (cm) & $N$ & Média & DP & Moda & $1^{\circ}$ quartil & $\begin{array}{l}2^{\circ} \text { quartil } \\
\text { (mediana) }\end{array}$ & $3^{\circ}$ quartil & $\begin{array}{c}\text { Percentil } \\
\text { de } 10 \%\end{array}$ & $\begin{array}{l}\text { Percentil } \\
\text { de } 90 \%\end{array}$ & Mínimo & Máximo \\
\hline AST & 95 & 1,58 & 0,46 & 1,4 & 1,3 & 1,5 & 1,9 & 1 & 2,3 & 0,5 & 2,8 \\
\hline PST & 95 & 2,32 & 0,46 & 2,3 & 2 & 2,3 & 2,6 & 1,8 & 2,9 & 1 & 3,7 \\
\hline FP & 95 & 1,04 & 0,43 & 1 & 0,8 & 1 & 1,3 & 0,5 & 1,54 & 0 & 2,5 \\
\hline LM anterior & 62 & 0,59 & 0,31 & 0,5 & 0,3 & 0,5 & 0,8 & 0,2 & 1,07 & 0,1 & 1,3 \\
\hline Posterior & 15 & 0,37 & 0,17 & 0,2 & 0,2 & 0,3 & 0,5 & 0,2 & 0,62 & 0,2 & 0,8 \\
\hline Central & 18 & 0 & & 0 & 0 & 0 & 0 & & & & \\
\hline
\end{tabular}

Distância em relação à região anterossuperior do trocânter.

Dos 95 espécimes estudados, $100 \%$ dos casos estão entre o valor mínimo e máximo. que foi de 0,5 a $2,8 \mathrm{~cm}$; $90 \%$ não ultrapassaram $2,3 \mathrm{~cm}$ (percentil $90 \%$ ). A distância mediana observada foi de $1,5 \mathrm{~cm}$ e a amplitude interquartílica (AIQ = Q3-Q1), de 0,6 cm.

\section{Distância em relação à região anteroposterior do trocânter}

Dos 95 espécimes estudados, $100 \%$ dos casos estão entre o valor mínimo e máximo, que foi de 1,0 a 3,7cm; $90 \%$ não ultrapassaram $2,9 \mathrm{~cm}$ (percentil 90\%). A distância mediana observada foi de $2,3 \mathrm{~cm}$ e a amplitude interquartílica $(\mathrm{AIQ}=\mathrm{Q} 3-\mathrm{Q} 1)$, de $0,6 \mathrm{~cm}$.

\section{Distância em relação à fossa piriforme}

Dos 95 espécimes estudados, $100 \%$ dos casos estão entre o valor mínimo e máximo, que foi de 0 a $2,5 \mathrm{~cm}$; $90 \%$ não ultrapassaram $1,54 \mathrm{~cm}$ (percentil 90\%). A distância mediana observada foi de $1,0 \mathrm{~cm}$ e a amplitude interquartílica (AIQ $=\mathrm{Q} 3-\mathrm{Q} 1)$, de $0,5 \mathrm{~cm}$, que foi a menor distância entre as regiões anatômicas considerados, expressando inicialmente, em relação à fossa piriforme, melhor precisão (Figura 3).

\section{DISCUSSÃO}

A haste intramedular anterógrada é um procedimento padrão para as fraturas da diáfise do fêmur, tanto nas fraturas fechadas como nas expostas ${ }^{(12,13)}$. Apesar de existirem os pontos de referência anatômicos na extremidade proximal do fêmur, o cirurgião pode encontrar alguma dificuldade em localizar o ponto de entrada ideal quando opta pelo tratamento com a haste intramedular. Muitos autores apontam a grande importância do ponto de entrada correto na haste intramedular anterógrada ${ }^{(1,14,15)}$; a escolha errada do local pode causar várias complicações

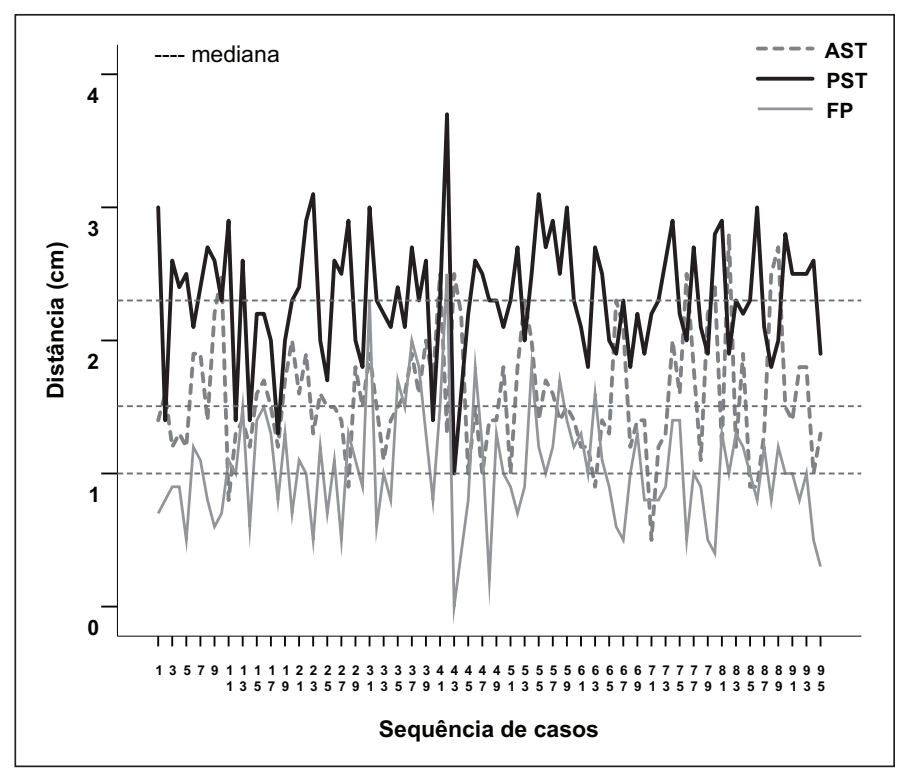

Fonte: Faculdade de Medicina de Petrópolis/RJ

Figura 3 - llustra a sequência dos pontos das distâncias segundo as regiões anatômicas. (AST - distância em relação à região anterossuperior do trocânter; PST - distância em relação à região anteroposterior do trocânter; FP- distância em relação à fossa piriforme).

intraoperatórias como deformidades angulares no pósoperatório $^{(5,14-19)}$. As informações sobre a correta localização do ponto de entrada são raramente encontradas na literatura e são controversas e confusas ${ }^{(2,9,20,21)}$.

$\mathrm{Na}$ descrição original de Küntscher, citado por Gausepohl et al ${ }^{(2)}$, ele somente menciona que o puncionador é colocado na ponta do trocânter maior sob o controle fluoroscópico. Christensen ${ }^{(21)}$ simplesmente mencionou que a ponta do trocânter, e não a fossa trocantérica, deveria ser usada para a inserção da haste. Outros autores recomendam que o ponto de entrada seja colocado sobre a face medial do trocânter maior ${ }^{(22,23)}$. Hansen e Winquist ${ }^{(24)}$ recomendaram um ponto entre a junção do colo femoral e o trocânter. Porém, não demonstraram a exata localização anatômica do ponto de entrada ou enfatizaram a posição no plano sagital. Kempf et $a l^{(1)}$ acreditam que o ponto de 
entrada ideal é a ponta do trocânter, porém, sugerem um ponto mais medial da parede do trocânter na fratura proximal do fêmur. Mais recentemente, vários autores têm recomendado um ponto de entrada medial e posterior ao trocânter maior, na fratura da extremidade proximal do fêmur ${ }^{(5,25,26)}$. Georgiadis et $a l^{(9)}$ utilizando somente a parte superior do fêmur, até o seu istmo, definiram como o ponto de entrada ideal sendo no trocânter maior, em uma posição mais dorsal, comparada à inserção tendinosa do músculo piriforme. Gausepohl et al ${ }^{(2)}$ incluíram em sua pesquisa o terço distal do fêmur, considerando a curvatura natural do fêmur; resultados demonstraram que o ponto de entrada ideal ficou significantemente mais ventral sobre a inserção do músculo piriforme.

Harper et $a l^{(27)}$ introduziram guias intramedulares de $3 \mathrm{~mm}$ de diâmetro de forma retrógrada na região intercondiliana do fêmur. Concluíram que a ponta do trocânter não foi a saída mais natural do guia e que o melhor local foi a junção entre o colo do fêmur e o trocânter. Nossos resultados, também utilizando um guia de $3 \mathrm{~mm}$ de diâmetro introduzido de forma retrógrada a $1,2 \mathrm{~cm}$ na região intercondiliana do fêmur, região considerada ser o centro do fêmur, demonstraram resultados relativamente uniformes. A saída natural do guia ficou na fossa piriforme, que demonstrou um intervalo menor (distân-

\section{REFERÊNCIAS}

1. Kempf I, Grosse A, Beck G. Closed locked intramedullary nailing. J Bone Joint Surg Am. 1985;67(5):709-20.

2. Gausepohl T, Pennig J, Koebke J, Harnoss S. Antegrade femoral nailing: an anatomical determination of the correct entry point. Injury. 2002;33(8):701-5.

3. Müller ME, Allgöwer M, Schneider R, Willenegger $H$. Manual de osteossíntese: técnicas recomendadas pelos Grupos AO-ASIF. Tradução de: Nelson Gomes de Oliveira. $3^{a}$ ed. São Paulo: Manole; 1993. p.151-8.

4. Kropfl A, Naglik H, Primavesi $\mathrm{C}$, Hertz H. Unreamed intramedullary nailing of femoral fractures. J Trauma. 1995;38(5):717-26.

5. Winquist RA, Hansen ST, Clawson DK. Closed intramedullary nailing of femoral fractures: a report of five hundred and twenty cases. J Bone Joint Surg Am. 1984;66(4):529-39.

6. Beaty JH, Austin SM, Warner WC, Nichols L. Interlocking intramedullary nailing of femoral-shaft fractures in adolescents: preliminary results and complications. J Pediatr Orthop. 1994;14(3):178-83.

7. Bednar DA, Ali P. Intramedullary nailing of femoral shaft fractures: reoperation and return to work. Can J Surg. 1993;36(5):464-6.

8. Bain GI, Zacest AC, Paterson DC, Middleton J, Pohl AP. Abduction strength following intramedullary nailing of the femur. J Orthop Trauma. 1997;11(2):93-7.

9. Georgiadis GM, Olexa TA, Ebraheim NA. Entry sites for antegrade femoral nailing. Clin Orthop Relat Res. 1996;(330):281-7.

10. Krupp RJ, Malkani AL, Goodin RA, Voor MJ. Optimal entry point for retrograde femoral nailing. J Orthop Trauma. 2003;17(2):100-5.

11. Carmack DB, Moed BR, Kingston C, Zmurko M, Watson JT, Richardson M. Identification of the optimal intercondylar starting point for retrograde femoral nailing: an anatomic study. J Trauma. 2003;55(4):692-5.

12. Buchholz RW, Jones A. Current concepts review: fractures of the shaft of the femur. J Bone Joint Surg Am. 1991;73(10):1561-5.

13. Wolinsky PR, McCarty E, Shyr Yu, Johnson KD. Reamed intramedullary nailing of the femur: 551 cases. J Trauma. 1999;46(3):392-9.

14. Browner BD. Pitfalls, errors, and complications in the use of locking Küntscher nails. Clin Orthop Relat Res. 1986;(212):192-208. cia mediana de $1,0 \mathrm{~cm}$ e a amplitude interquartílica de $0,5 \mathrm{~cm})$. Isso significa melhor precisão e provavelmente se reproduz com melhor confiabilidade.

A curva de raio do fềmur deve ser considerada quando se opta pelo tratamento das fraturas do fêmur com a haste intramedular. O ponto de entrada neutro pode ser obtido, iniciando o seu posicionamento na fossa trocantérica ou na ponta do trocânter, não mais que $2 \mathrm{~cm}$ da região posterior do trocânter ${ }^{(9)}$. Observamos que no posicionamento do fio-guia, em relação à região anteroposterior proximal do fêmur, $62 \%$ tiveram a saída anterior à linha média do fêmur. Isso pode ter sido devido à utilização de um fioguia reto, não acompanhando a curva de raio do fêmur, o que pode ser um fator de crítica. Harper et al ${ }^{(27)}$ introduziram implantes com curva de raio $(203 \mathrm{~cm}$ e $137 \mathrm{~cm})$ e encontraram um ponto de entrada localizado dorsal ao trocânter, resultado diferente do encontrado quando utilizaram fio-guia reto, que ficou mais anterior.

\section{CONCLUSÃO}

O eixo central do canal medular, na incidência coronal, projetou melhor precisão na região da fossa piriforme. Para hastes retas, a melhor localização do ponto de entrada é a fossa piriforme.

15. Johnson KD, Tencer AF, Sherman MC. Biomechanical factors affecting fracture stability and femoral bursting in closed intramedullary nailing of femoral shaft fractures, with illustrative case presentations. J Orthop Trauma. 1987;1(1):1-11

16. Astion DJ, Wilber JH, Scoles PV. Avascular necrosis of the capital femoral epiphysis after intramedullary nailing for a fracture of the femoral shaft. J Bone Joint Surg Am. 1995;77(7):1092-4.

17. Brumback RJ, Wells JD, Lakatos R, Poka A, Bathon GH, Burgess AR. Heterotopic ossification about the hip after intramedullary nailing for fractures of the femur. J Bone Joint Surg Am. 1990;72(7):1067-73.

18. Miller SD, Burkart B, Damson E, Shrive N, Bray RC. The effect of the entry hole of an intramedullary nail on the strength of the proximal femur. J Bone Joint Surg Br. 1993;75(2):202-6.

19. Thometz JG, Lamdan R. Osteonecrosis of the femoral head after intramedullary nailing of a fracture of the femoral shaft in an adolescent: a case report. J Bone Joint Surg Am. 1995;77(9):1423-6.

20. Dora C, Leunig M, Beck M, Rothenfluh D, Ganz R. Entry point soft tissue damage in antegrade femoral nailing: a cadaver study. J Orthop Trauma. 2001;15(7):488-93.

21. Christensen NO. Technique, errors and safeguards in modern Küntscher nailing. Clin Orthop Relat Res. 1976;(115):182-8.

22. Böhler J. Closed intramedullary nailing of the femur. Clin Orthop Relat Res. 1968;(60):51-67.

23. Rascher JJ, Nahigian SH, Macys JR, Brown JE. Closed nailing of femoral shaft fractures. J Bone Joint Surg Am. 1972;54(3):534-44.

24. Hansen ST, Winquist RA. Closed intramedullary nailing of the femur. Clin Orthop Relat Res. 1979;(138):56-61

25. Kyle RF. Fractures of the proximal part of the femur. J Bone Joint Surg Am. 1994;76(6):924-50.

26. Winquist RA. Locked femoral nailing. J Am Acad Orthop Surg. 1993;1(2):95-105.

27. Harper MC, Carson WL. Curvature of the femur and the proximal entry point for an intramedullary rod. Clin Orthop Relat Res. 1987;(220):155-61. 